\title{
SUR QUELQUES ESPÈCES PEU COMMUNES \\ DU GENRE IXODES (ACARIENS, IXODIDAE)
}

\author{
Par P.-C. MOREL
}

Je signale ici des références nouvelles concernant certains Ixodes d'Europe, assez rares pour que ces références présentent malgré tout quelque intérêt. Parmi ces espèces, certaines ont déjà fait l'objet de révision et leur statut systématique est bien établi. Pour d'autres nous utiliserons les noms qui paraissent le mieux convenir, sous réserve de modifications à venir lorsque des collections importantes nous permettront de nous faire une opinion sur leur validité ; c'est le cas pour beaucoup des Ixodes décrits par Schulze. Les espèces mentionnées ici ont été récoltées personnellement, ou examinées dans diverses collections françaises: collection Brumpt (Faculté de Médecine de Paris) : CBpt ; collection du Muséum d'Histoire naturelle de Paris (laboratoire du $\mathrm{M}^{\mathrm{r}}$ Marc André) : MHNP ; collection Neumann à l'Ecole vétérinaire de Toulouse : CNm ; collection R.-Ph. Dollfus (Inst. Hautes-Etudes).

IXODES ACUMINATUS Neumann, 1901.

1 $3 \mathrm{n} 61$ Rennes (9-IV-20) : hérisson (CBpt : E. Brumpt, legit) ; référence publiée par Warburton (1926), mais omise par Sénevet (1937).

$1 \% 1 \mathrm{n}$ Padulone (Corse): Crocidura cyrnensis (25-IV-26) (CBpt).

IXODES CANISUGA Johnston, 1849.

4 요 Toulouse : chien (CNm 734) (= Neumann, 1901).

2 우 Fontainebleau : blaireau (CNm 735 : ex-coll. Railliet) (= Neumann, 1901).

4 ㅇํ Forêt de Lancôme (Indre) : chien (IV-1912) (CBpt).

Il s'agit de I. canisuga sensu lato; les caractères que donne Schulze pour distinguer les diverses espèces au sein de ce groupe paraissent difficiles à apprécier sur quelques exemplaires; il est d'ailleurs possible que ces espèces ne soient en fait que des variations individuelles.

Ann. de Parastrologie, T. XXXIII, N $4,1959$. 
IXODES FRONTALIS Panzer, 1795.
1 ㅇ
Challans (Vendée) (II-45): libre (MHNP : Dresco legit).
1 Concarneau (Fin.) (IX-21) : Regulus regulus (R.-Ph. Dollfus legit, coll. Dollfus).
$3 \mathrm{n} \quad$ Gif-sur-Yvette (S.-et-O.) (VI-53): Parus ater (ipse legi).
$2 \mathrm{n} \quad$ Boiscorbon (S.-et-().) (IV-54) : Turdus musicus (ipse legi).

IXODES LIVIDUS Koch, 1884, sensu Schulze \& Schlottke, 1929.

$1+$ Rouen : Riparia riparia (11-VIII-83) (CBpt: Gadeau de Kervil!e legit).

L'hirondelle de rivage est l'hôte spécifique de cet Ixodes, bien différent de $I$. canisuga (notamment par la dentition de l'hypostome), avec lequel il a parfois été confondu. Ce qui est moins sûr, c'est le nom exact qui doit lui être appliqué. Schulze a également utilisé le terme d'I. plumbeus Leach, 1815 ( $\neq$ plumbeus $\mathrm{F} ., 1805$ ).

IXODES ROTSCHILDI Nuttall \& Warburton, 1911.

2 우 Morothoe (Gde-Bretagne) : macareux (CNm 1362 : exBritish Museum of Natural History) (déterminés I. frontalis par Neumann).

IXODES STRIGICOLA Schulze \& Schlottke, 1929.

$2 \mathrm{n}$
Bures-sur-Yvette (S.-et-O.) (V-53): Athene noctua (ipse legi).

J'utilise le terme strigicola, car mes exemplaires ne correspondent pas au dessin de la nymphe de passericola Schulze, 1933, dans Arthur (1952); en utilisant les clés de Schulze (1937), en supposant une certaine communauté de caractères entre les femelles et les nymphes, j'arrive au complexe arboricola-strigicola-dryadis ; il est très probable que ces espèces n'en sont qu'une seule ; je choisis le nom de strigicola en raison de l'hôte sur lequel les tiques ont été prélevées, tout en sachant bien que cette détermination n'est que provisoire. 
IXODES (CERATIXODES) URIAE White, 1852.

$1 \mathrm{n}$ Ile Ricard (Roscoff, Fin.) (27-V-25) : macareux (CBpt).

C'est la première fois que l'espèce est mentionnée en France. En fait, on doit pouvoir la trouver dans toutes les nicheries d'oiseaux marins de la côte bretonne (en même temps peut-être que 1 . rotschildi Nuttall et Warburton, 1911 et I. unicavatus Neumann, 1908 qui n'ont pas encore été signalés de France). Autre référence :

Beluchtcha-Bay (Nouvelle-Zemble) (MHNP : VII-1908, Mission aretique française Kostin-Charcot).

\section{BIBLIOGRAPHIE}

ARthUR (D. R.) (1952). - Ixodes passericola Schulze. A description of the hitherto unknown male and larva, with a redescription of the femae and nymph. Parasit., 42, (1-2) : 155-159.

- (1953). - The systematic status of Ixodes percavatus var. rotschildi Nuttall et Warburton, 1911. Parasit., 13, (3-4) : 222-226.

Schulze (P.) (1937). - Die kleinhöhlenbewohnenden Zecken der Artengruppe um Ixodes autumnalis Leach, 1815. Z. Parazitenk., 9, (3) : 351-372.

- et Schцоттке (E.) (1929), - Kleinhöhlenbewohnenden deutsche Zecken, mit Beschreibung dreier neuer Baumhohlenbrüter und einer Bestimmungstabelle der deutschen Ixodes. Sitz. Ber. Ges. Naturf. Rostock, ser. $3,2: 95-110$.

Senevet (G.) (1937), - Ixodoides. Fanne de France, Paris (Lechevalier édit.), $101 \mathrm{pp}$. 